\title{
A COMPARISON OF ADAPTIVE BEAMFORMING IMPLEMENTATIONS FOR WIDEBAND SCENARIOS
}

\author{
Stephan Weiss*, Choo Leng Koh* and Wei Liu ${ }^{\dagger}$ \\ * Communications Research Group, School of ECS, University of Southampton, UK \\ $\dagger$ Department of Electronic and Electrical Eng., University of Sheffield, Sheffield, UK
}

Keywords: Broadband beamforming, adaptive signal processing, efficient implementation, subband processing, frequency domain processing.

\begin{abstract}
In this paper, we compare low cost implementations of wideband adaptive beamformers utilising the generalised sidelobe canceller (GSC) in combination with the least-mean squares algorithms. DFT based techniques suffer from unjustified narrowband assumptions. We therefore derive and investigate an overlap-save implementation of the GSC, which offers a steady state suppression of jammers equivalent to a time domain GSC but may be prone to slow convergence. Finally, subband techniques offer a more robust convergence trade-off for a somewhat higher computational cost. Analysis and simulation results revealing some of the algorithms' properties are presented.
\end{abstract}

\section{Introduction}

Ultra-wideband (UWB) communications offers a radically different approach to wireless communication compared to conventional narrowband systems. This fast emerging technology with uniquely attractive features invite major advances in wireless communications, networking, radar, imaging and positioning systems [1]. However, for this technology to achieve high spectral and spatial resolution, the array usually requires a considerable temporal and spatial dimension. Thus, a larger number of sensors, $M$, and tap delay line (TDL) elements, $L$, results in a very large computational complexity of wideband beamforming algorithms, inhibiting the deployment of powerful techniques such as recursive least squares (RLS) algorithms.

To reduce the computational complexity of wideband beamforming algorithms, transformations can be applied to the TDL elements of each sensor. For example, the discrete Fourier transform (DFT) yields frequency bins, on which independent narrowband beamforming algorithms can be performed $[3,4$, 5], yielding tremendous savings [6]. However, the suboptimality of these narrowband solutions with respect to the wideband problem, as established in e.g. $[4,7,8]$, requires sliding window and block processing implementations [5] to be replaced by algorithms based on overlap-add or overlap-save techniques $[8,9,10]$. This step enhances the mean square error

This work was supported by the U.K. MoD's Corporate Research Programme. performance, but increases the computational load compared to sliding window and block processing. Further, subband transformations for adaptive filtering [11] can be applied to wideband beamforming $[12,13,14]$. There, filter banks with a high frequency selectivity produce non-critically decimated aliasfree subband signals, which can be processed independently. Although the various subbands will require wideband beamforming algorithms, a considerably reduced temporal dimension can be achieved [7, 13].

This paper performs a comparative study of the various methods - in terms of computational cost, convergence speed, and steady-state error - by example of the generalised sidelobe canceller (GSC) [2, 15]. Sec. 2 briefly reviews the GSC's structure, while the various low-complexity realisations are discussed in Sec. 3. Simulations in Sec. 4 will provide insight into the performance of various systems, and conclusions be drawn in Sec. 5.

\section{Wideband Beamforming via the Generalised Sidelobe Canceller}

We consider the wideband beamforming structure depicted in Fig. 1, which acquires a spatio-temporal waveform by means of $M$ sensor signals $x_{m}[n], m=0(1) M-1$ and fed into tappeddelayed lines (TDLs) of dimension $L$. At each discrete time instance $n$, an $M L$ dimensional data vector

$$
\begin{aligned}
\mathbf{x}[n] & =\left[\mathbf{x}_{0}^{\mathrm{T}}[n] \mathbf{x}_{1}^{\mathrm{T}}[n] \ldots \mathbf{x}_{M-1}^{\mathrm{T}}[n]\right]^{\mathrm{T}} \\
\mathbf{x}_{m}[n] & =\left[x_{m}[n] x_{m}[n-1] \ldots x_{m}[n-L+1]\right]^{\mathrm{T}}
\end{aligned}
$$

is passed to the beamforming processor. We consider a linear wideband beamformer, where a a linear output

$$
e[n]=\mathbf{w}^{\mathrm{H}} \mathbf{x}[n],
$$

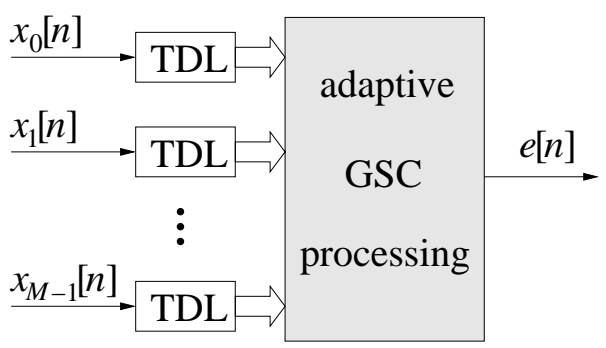

Fig. 1. Time-domain beamforming structure. 
is formed by a scalar product with the beamforming weights in $\mathbf{w}$,

$$
\begin{aligned}
\mathbf{w} & =\left[\begin{array}{llll}
\mathbf{w}_{0}^{\mathrm{T}} & \mathbf{w}_{1}^{\mathrm{T}} & \cdots & \mathbf{w}_{M-1}^{\mathrm{T}}
\end{array}\right]^{\mathrm{T}} \\
\mathbf{w}_{m} & =\left[\begin{array}{llll}
w_{m, 0} & w_{m, 1} & \cdots & w_{m, J-1}
\end{array}\right]^{\mathrm{T}}
\end{aligned}
$$

Exemplarily, we want to consider the linearly constrained minimum variance (LCMV) beamforming problem [19],

$$
\min _{\mathbf{w}} \mathbf{w}^{\mathrm{H}} \mathbf{R}_{x x} \mathbf{w} \quad \text { subject to } \quad \mathbf{C}^{\mathrm{H}} \mathbf{w}=\mathbf{f} \quad
$$

where $\mathbf{R}_{x x}=\mathcal{E}\left\{\mathbf{x}[n] \mathbf{x}^{\mathrm{H}}[n]\right\}$ is the covariance matrix, $\mathbf{C} \in$ $\mathbb{C}^{M J \times r}$ the constraint matrix and $\mathbf{f} \in \mathbb{C}^{r}$ the response vector for linearly independent constraints.

The generalised sidelobe canceller (GSC) transforms (5) into an unconstrained optimisation problem by projecting the $M L$ element data vector by a blocking matrix $\mathbf{B} \in \mathbb{C}^{M L \times M L-r}$ onto a subspace independent of the constraints, i.e. $\mathbf{u}[n]=$ $\mathbf{B}^{\mathrm{H}} \mathbf{x}[n]$, and by a quiescent vector $\mathbf{w}_{\mathrm{q}}$ to obtain the signal of interest plus interference, $d[n]=\mathbf{w}_{\mathrm{q}}^{\mathrm{H}} \mathbf{x}[n]$. Unconstrained optimisation of the output

$$
e[n]=d[n]-\mathbf{w}_{\mathrm{a}}^{\mathrm{H}} \mathbf{u}[n]
$$

can then be performed to linearly combine the blocking matrix outputs by coefficients in $\mathbf{w}_{\mathrm{a}} \in \mathbb{C}^{M L-r}$ to minimise any interference remaining in $d[n]$ in the mean square error (MSE) sense $[2,15]$. With the projections amounting to $(M L)^{2}$ multiply-accumulates (MACs), the optimisation can be performed, for example, by a normalised LMS algorithm with a complexity of approximately $3 M L$ MACs.

\section{Efficient Implementations}

\subsection{Independent Frequency Bin Processing}

A class of popular beamforming algorithms applies DFTs to each TDL, and independently processes the resulting frequency bins by $L$ narrowband beamforming algorithms, e.g. a GSC, as shown in Fig. 2, with $\mathbf{H}(z)$ representing an $L$-point DFT matrix $[3,4,5]$. The resulting cost for processing the signal in blocks of $L$ samples accrues to

$$
\mathcal{C}_{\text {fb }, \text { block }}=(M+1) \log _{2} L+M^{2}+3 M
$$

complex MACs per fullband sampling period. A sliding window version of this algorithm computes the DFTs at each time instance $n$, and replaces the IDFT at the beamformer output in Fig. 2 by a simple summation [5], yielding a slightly higher cost of

$$
\mathcal{C}_{\mathrm{fb}, \text { sliding }}=M L \log _{2} L+L M^{2}+3 L M
$$

MACs per fullband sampling period.

In both block and sliding window DFT implementations, we use a vector $\mathbf{x}_{\mathrm{fd}}$ to denote the input data to the GSC beamformer.

$$
\mathbf{x}_{\mathrm{fd}}[n]=\mathbf{P}\left[\begin{array}{ccc}
\mathbf{T}_{L} & & \mathbf{0} \\
& \ddots & \\
\mathbf{0} & & \mathbf{T}_{L}
\end{array}\right] \cdot \mathbf{x}[n]
$$

where $\mathbf{T}_{L}$ is an $L$-point DFT matrix applied to each TDL and $\mathbf{P}$ a permutation matrix such that $\mathbf{x}_{\mathrm{fd}}[n]$ is ordered w.r.t. frequency bins. The latter vector contains the $M$ sensor components for the first frequency bin in its first $M$ elements, and so forth until the last $M$ elements are occupied by the $M$ sensor components of the $L$ th frequency bin.

By applying a DFT to the constraint equation in (5), constraints for sliding window and block processors can be derived. If the signal of interest illuminates the array from broadside, the $r=L$ constraints are decoupled and identical for every frequency bin. As a result, the input to the adaptive GSC processor can be denoted as

$$
\mathbf{u}_{\mathrm{fd}}[n]=\underbrace{\left[\begin{array}{ccc}
\mathbf{B}^{\mathrm{H}} & & \mathbf{0} \\
& \ddots & \\
\mathbf{0} & & \mathbf{B}^{\mathrm{H}}
\end{array}\right]}_{\mathbf{B}_{\mathrm{fd}}^{\mathrm{H}}} \mathbf{x}_{\mathrm{fd}}[n]
$$

with $\mathbf{u}_{\mathrm{fd}}[n] \in \mathbb{C}^{(M-1) L}$ and $\mathbf{B} \in \mathbb{C}^{M \times M-1}$. Note that the blocking matrix $\mathbf{B}$ applied to each frequency bin is different from the blocking matrix defined in Sec. 2. Therefore, the covariance matrix $\mathbf{R}_{\mathbf{u u}, \mathrm{fd}}=\mathcal{E}\left\{\mathbf{u}_{\mathrm{fd}}[n] \cdot \mathbf{u}_{\mathrm{fd}}^{\mathrm{H}}[n]\right\}$ is given by

$$
\mathbf{R}_{u u, \mathrm{fd}}=\mathbf{B}_{\mathrm{fd}}^{\mathrm{H}}\left[\begin{array}{cccc}
\mathbf{R}_{0,0} & \mathbf{R}_{1,0} & \ldots & \mathbf{R}_{L-1,0} \\
\mathbf{R}_{0,1} & \mathbf{R}_{1,1} & \ldots & \mathbf{R}_{L-1,1} \\
\vdots & \vdots & \ddots & \vdots \\
\mathbf{R}_{0, L-1} & \mathbf{R}_{1, L-1} & \ldots & \mathbf{R}_{L-1, L-1}
\end{array}\right] \mathbf{B}_{\mathrm{fd}}
$$

where $\mathbf{R}_{i, j}$ are $M \times M$ correlation matrices between frequency bins $i$ and $j$ of the different sensor signals prior to passing the blocking matrix $\mathbf{B}_{\mathrm{fd}}$.

Block and sliding window processing neglect any correlation between frequency bins. With this approximation, the covariance matrix to the resulting independent frequency bin (IFB) processor $\mathbf{R}_{u u, f d}$ is forced to attain the form

$$
\mathbf{R}_{u u, \text { ifb }}=\left[\begin{array}{cclc}
\mathbf{B}^{\mathrm{H}} \mathbf{R}_{0,0} \mathbf{B} & \mathbf{O}_{L} & \ldots & \mathbf{O}_{L} \\
\mathbf{O}_{L} & \mathbf{B}^{\mathrm{H}} \mathbf{R}_{1,1} \mathbf{B} & \ldots & \mathbf{O}_{L} \\
\vdots & \vdots & \ddots & \vdots \\
\mathbf{O}_{L} & \mathbf{O}_{L} & \ldots & \mathbf{B}^{\mathrm{H}} \mathbf{R}_{L-1, L-1} \mathbf{B}
\end{array}\right],
$$

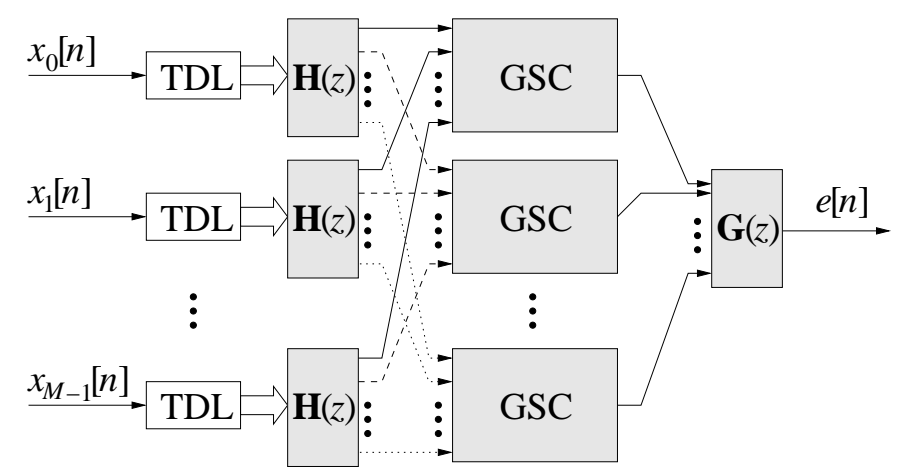

Fig. 2. Transform domain beamforming structure. 
i.e. any correlation between bins $i$ and $j \neq i$ is neglected. However, due to the high sidelobes in the DFT's frequency response characteristics as depicted in Fig. 3, adaptive algorithms suffer from spectral leakage — limiting their convergence [16] and potentially need a substantial amount of degrees of freedom to approximately suppress even low rank interferers [7]. Recently, such failures have generally been attributed to the application of essentially narrowband processing to wideband problems [8].

\subsection{Overlap-Save DFT Implementation}

In order to overcome the problem of inter-frequency bin correlations being neglected, overlap-add or overlap-save methods can be applied to accurately address wideband problems in the DFT-domain $[8,9,10]$. Overlap-add and overlap-save techniques exploit the Toeplitz nature of the of data matrix, transforming it to a circulant form by increasing the DFT to $2 L$ points. Unlike the circular convolution between the signals $x_{m}[n]$ and the filters following each sensor implemented by the previously outlined methods, they now realise a linear convolution [9]. By rigorously minimising time domain criteria, such as the mean square error, expressed in the DFT domain, exact wideband DFT domain methods can be attained and, if required, subsequently simplified [8].

\subsubsection{Exact Frequency Domain Formulation}

We first introduce a block processing notation by stacking the outputs $e^{*}[n L], e^{*}[n L+1], \cdots, e^{*}[n L+L-1]$ into a vector $\mathbf{e}[n] \in \mathbb{C}^{L}$, yielding

$$
\mathbf{e}[n]=\sum_{m=0}^{M-1} \mathbf{X}_{m}^{\mathrm{H}}[n] \mathbf{w}_{m}
$$

where with (2)

$$
\mathbf{X}_{m}[n]=\left[\mathbf{x}_{m}[n L] \mathbf{x}_{m}[n L+1] \cdots \mathbf{x}_{m}[n L+L-1]\right] .
$$

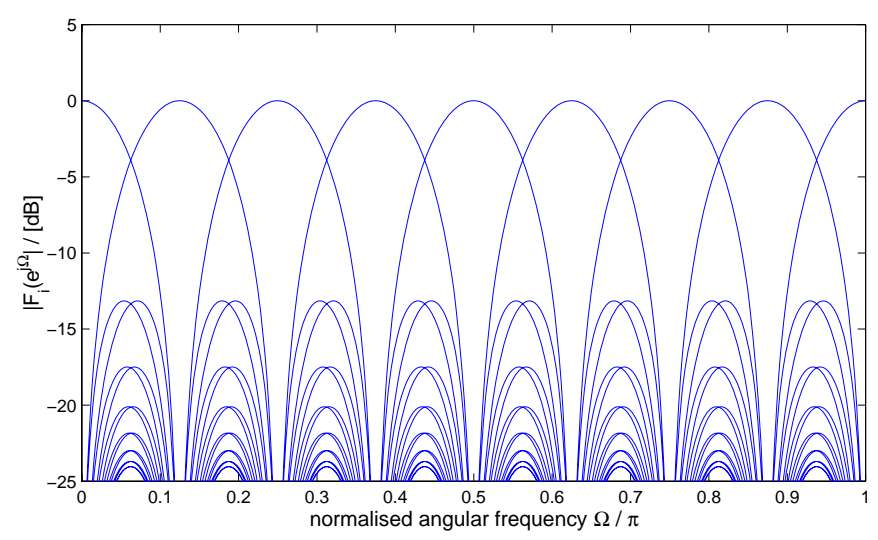

Fig. 3. Filter bank characteristic of a 16-point DFT.
We can expand the convolutional matrices $\mathbf{X}_{m}[n]$ to a circulant form [20] and write the output of the beamformer as

$$
\left[\begin{array}{c}
\mathbf{v} \\
\mathbf{e}[n]
\end{array}\right]=\sum_{m=0}^{M-1}\left[\begin{array}{cc}
\tilde{\mathbf{X}}_{m}^{\mathrm{H}}[n] & \mathbf{X}_{m}^{\mathrm{H}}[n] \\
\mathbf{X}_{m}^{\mathrm{H}}[n] & \tilde{\mathbf{X}}_{m}^{\mathrm{H}}[n]
\end{array}\right] \cdot\left[\begin{array}{c}
\mathbf{w}_{m} \\
\underline{\mathbf{0}}
\end{array}\right]
$$

where $\mathbf{v}$ is an arbitrary $J$ element vector and $\tilde{\mathbf{X}}_{m}$ is a Toplitz matrix using the data samples of $\mathbf{X}_{m}$ except for an arbitrary element along the main diagonal. Note that with a $2 L$ point DFT matrix $\mathbf{T}$, the circulant property can be exploited such that

$$
\begin{aligned}
\underline{\underline{\Gamma}}_{m}[n] & =\mathbf{T} \cdot\left[\begin{array}{cc}
\tilde{\mathbf{X}}_{m}^{\mathrm{H}}[n] & \mathbf{X}_{m}^{\mathrm{H}}[n] \\
\mathbf{X}_{m}^{\mathrm{H}}[n] & \tilde{\mathbf{X}}_{m}^{\mathrm{H}}[n]
\end{array}\right] \cdot \mathbf{T}^{\mathrm{H}} \\
& =\operatorname{diag}\left\{\mathbf{T J}\left[\begin{array}{c}
\mathbf{x}_{m}[n L+L] \\
\mathbf{x}_{m}[n L]
\end{array}\right]\right\}
\end{aligned}
$$

takes on a diagonal form [20], whereby $\mathbf{J}$ is a reverse identity. Therefore, the definition of a DFT domain error vector $\underline{\mathbf{e}}[n] \in$ $\mathbb{C}^{2 L}$ leads to

$$
\begin{aligned}
\underline{\mathbf{e}}[n] & =\mathbf{T}\left[\begin{array}{c}
\underline{0} \\
\mathbf{e}_{n}
\end{array}\right] \\
& =\underbrace{\mathbf{T}\left[\begin{array}{cc}
\mathbf{0}_{L \times L} & \mathbf{0}_{L \times J} \\
\mathbf{0}_{L \times L} & \mathbf{I}_{L}
\end{array}\right] \mathbf{T}^{\mathrm{H}}}_{\mathbf{G}} \cdot \mathbf{T}\left[\begin{array}{c}
\mathbf{v} \\
\mathbf{e}_{n}
\end{array}\right] \\
& =\mathbf{G} \sum_{m=0}^{M-1} \underline{\boldsymbol{\Gamma}}_{m}[n] \cdot \mathbf{T}\left[\begin{array}{c}
\mathbf{w}_{m} \\
\underline{\mathbf{0}}
\end{array}\right]=\mathbf{G} \underline{\underline{\mathbf{\Gamma}}}[n] \underline{\mathbf{w}}
\end{aligned}
$$

in dependency of the frequency domain coefficients $\underline{\mathbf{w}} \in \mathbb{C}^{2 M L}$ with $\mathbf{G} \in \mathbb{C}^{2 L \times 2 L}$, and $\underline{\underline{\boldsymbol{\Gamma}}}[n] \in \mathbb{C}^{2 L \times 2 M L}$.

To formulated the constraint equation with the frequency domain variables,

$$
\mathbf{C}^{\mathrm{H}} \mathbf{w}=\sum_{m=0}^{M-1} \mathbf{C}_{m}^{\mathrm{H}} \cdot \mathbf{w}_{m}=\mathbf{f}
$$

whereby the original matrix equation can be separated into $M$ additive components. Note that $\mathbf{C}_{m} \in \mathbb{C}^{L \times r}$ has an arbitrary form (in particular not Toeplitz), where $r$ is the number of linearly independent constraints. In terms of the DFT domain coefficients, we have

$$
\sum_{m=0}^{M-1}\left[\begin{array}{ll}
\mathbf{C}_{m}^{\mathrm{H}} & \mathbf{0}_{r \times J}
\end{array}\right]\left[\begin{array}{c}
\mathbf{w}_{m} \\
\underline{\mathbf{0}}
\end{array}\right]=\mathbf{f}
$$

or

$$
\sum_{m=0}^{M-1}\left[\begin{array}{ll}
\mathbf{C}_{m}^{\mathrm{H}} & \mathbf{0}_{r \times J}
\end{array}\right] \mathbf{T}^{\mathrm{H}} \underline{\mathbf{w}}_{m}=\underline{\underline{\mathbf{C}}}^{\mathrm{H}} \underline{\mathbf{w}}=\mathbf{f},
$$

where $\underline{\underline{\mathbf{C}}} \in \mathbb{C}^{2 M L \times r}$ is the new constraint matrix

$$
\underline{\underline{\mathbf{C}}}=\left[\left[\begin{array}{c}
\mathbf{C}_{0} \\
\mathbf{0}_{L \times r}
\end{array}\right]^{\mathrm{H}} \mathbf{T}^{\mathrm{H}} \ldots\left[\begin{array}{c}
\mathbf{C}_{M-1} \\
\mathbf{0}_{L \times r}
\end{array}\right]^{\mathrm{H}} \mathbf{T}^{\mathrm{H}}\right]^{\mathrm{H}}
$$

applicable to the DFT domain coefficient vector. 


\subsubsection{Overlap-Save GSC}

The LCMV formulation equivalent to the time domain but using frequency domain variables is now given by

$$
\min _{\underline{\mathbf{w}}} \mathcal{E}\left\{\underline{\mathbf{e}}^{\mathrm{H}}[n] \underline{\mathbf{e}}[n]\right\}=\min _{\underline{\mathbf{w}}} \underline{\mathbf{w}}^{\mathrm{H}} \mathbf{R} \underline{\mathbf{w}},
$$

subject to $\underline{\underline{\mathbf{C}}}^{\mathrm{H}} \underline{\mathbf{w}}=\mathbf{f}$, where $\mathbf{R}=\mathcal{E}\left\{\underline{\underline{\boldsymbol{\Gamma}}}[n]^{\mathrm{H}} \mathbf{G}^{\mathrm{H}} \mathbf{G} \underline{\underline{\boldsymbol{\Gamma}}}[n]\right\}=$ $\mathcal{E}\left\{\underline{\underline{\boldsymbol{\Gamma}}}[n]^{\mathrm{H}} \mathbf{G} \underline{\underline{\boldsymbol{\Gamma}}}[n]\right\}$.

Analogously to the time domain formulation of the GSC, the weight vector $\underline{w}$ is separated into two orthogonal components, $\underline{\mathbf{w}}=\underline{\mathbf{w}}_{\mathrm{q}}-\underline{\mathbf{v}}$, with a quiescent vector $\underline{\mathbf{w}}_{\mathrm{q}}$ representing a projection onto the constraints, and a projection away from the constraints, $-\underline{\mathbf{v}}$, given by

$$
\underline{\mathbf{v}}=\underline{\underline{B w}}_{\mathrm{a}}
$$

utilising the blocking matrix $\underline{\underline{B}}=\operatorname{span}\left\{\underline{\underline{C}}^{\perp}\right\}$, which spans the nullspace of the constraint matrix $\underline{\underline{C} \text {. }}$

Therefore, with (18), the beamformer output is given by

$$
\underline{\mathbf{e}}[n]=\mathbf{G} \underline{\underline{\boldsymbol{\Gamma}}}[n]\left(\underline{\mathbf{w}}_{\mathrm{q}}-\underline{\underline{\mathbf{B w}}}_{\mathrm{a}}\right) .
$$

Analogously to a time domain LMS algorithm, by using the instantaneous squared error as a cost function $\xi=\underline{\mathbf{e}}^{\mathrm{H}}[n] \underline{\mathbf{e}}[n]$ we obtain a stochastic gradient

$$
\begin{aligned}
\hat{\nabla} \xi & =\frac{\partial \xi}{\partial \underline{\mathbf{w}}_{\mathrm{a}}^{*}} \\
& =-\underline{\underline{\mathbf{B}}}^{\mathrm{H}} \underline{\underline{\Gamma}}^{\mathrm{H}}[n] \underline{\mathbf{e}}[n]
\end{aligned}
$$

where $\mathbf{G}^{\mathrm{H}} \mathbf{G}=\mathbf{G}$ has been exploited. The update equation for $\underline{\mathbf{w}}_{\mathrm{a}}$ can then be written as

$$
\underline{\mathbf{w}}_{\mathrm{a}}[n+1]=\underline{\mathbf{w}}_{\mathrm{a}}[n]+\mu \underline{\underline{\mathbf{B}}}^{\mathrm{H}} \underline{\boldsymbol{\Gamma}}^{\mathrm{H}}[n] \underline{\mathbf{e}}[n]
$$

The computational cost of this exact frequency domain GSC algorithm has been addressed in [22] and accrues to

$$
\mathcal{C}_{\text {fd,os }}=2 M\left(4 M L-2 r+\log _{2} L+3\right)+4 \log _{2} L+6
$$

complex MACs per fullband sampling period.

\subsubsection{Covariance Matrix}

To determine the input covariance matrix to the adaptive process, we follow a proof of convergence in the mean similar to the LMS algorithm [21]. If the MMSE solution is subtracted both sides of (27), it can be shown that

$$
\begin{aligned}
\underline{\tilde{w}}_{\mathrm{a}}[n+1] & =\underline{\tilde{w}}_{\mathrm{a}}[n]-\mu \underline{\underline{\mathbf{B}}}^{\mathrm{H}} \mathcal{E}\left\{\underline{\underline{\boldsymbol{\Gamma}}}^{\mathrm{H}}[n] \mathbf{G} \underline{\underline{\boldsymbol{\Gamma}}}[n]\right\} \underline{\underline{\mathbf{B}}} \underline{\tilde{w}}_{\mathrm{a}}[n] \\
& =\left(\mathbf{I}-\mu \underline{\underline{\mathbf{B}}}^{\mathrm{H}} \mathcal{E}\left\{\underline{\underline{\boldsymbol{\Gamma}}}^{\mathrm{H}}[n] \mathbf{G} \underline{\underline{\boldsymbol{\Gamma}}}[n]\right\} \underline{\underline{\mathbf{B}}}\right) \underline{\tilde{w}}_{\mathrm{a}}[n](29)
\end{aligned}
$$

with $\underline{\tilde{w}}_{\mathrm{a}}[n]=\mathcal{E}\left\{\underline{\mathbf{w}}_{\mathrm{a}}[n]-\underline{\mathbf{w}}_{\mathrm{a}, \mathrm{opt}}\right\}$, where $\underline{\mathbf{w}}_{\mathrm{a}, \mathrm{opt}}$ is the MMSE solution. If the solution in (29) is compared to the structurally identical derivation for the LMS, the term

$$
\mathbf{R}_{\mathrm{os}}=\underline{\underline{\mathbf{B}}}^{\mathrm{H}} \mathcal{E}\left\{\underline{\underline{\boldsymbol{\Gamma}}}^{\mathrm{H}}[n] \mathbf{G} \underline{\underline{\boldsymbol{\Gamma}}}[n]\right\} \underline{\underline{\mathbf{B}}} \in \mathbb{C}^{2 M L-r \times 2 M L-r}
$$

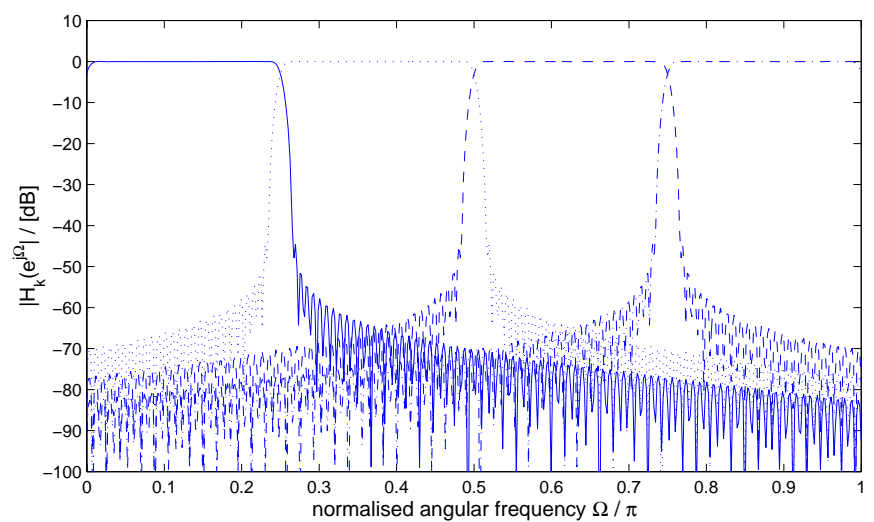

Fig. 4. Filter bank characteristic $(K=8, N=6)$.

can be identified as the covariance matrix in the overlap-save frequency domain implementation of the GSC. Note that no approximation have been made in the derivation of the overlapsave method with respect to the original time domain MSE, and that $\mathbf{R}_{\mathrm{os}}$ is not forced to be of block diagonal form as in the standard frequency domain approach of Sec. 3.1.

\subsection{Independent Subband Processing}

In subband adaptive beamforming, the blocks $\mathbf{H}(z)$ in Fig. 2 represent $K$-channel analysis filter banks which are non-critically decimated by a factor $N<K$. These filter banks can be derived from a real value prototype FIR filter by a generalised discrete Fourier transform (GDFT), according to [17]. The redundancy of the filter banks can be exploited to attain a high frequency selectivity where sidelobes between adjacent bins are kept to a minimum as illustrated in Fig. 4.

Due to the high sidelobe attenuation, the resulting subbands only overlap with adjacent bands and possess a sufficiently high stopband attenuation elsewhere. The covariance matrix of the filter bank outputs, given by $\mathbf{R}_{x x \text {,sub }}=\mathcal{E}\left\{\mathbf{x}_{\text {sub }} \cdot \mathbf{x}_{\text {sub }}^{H}\right\} \in$ $\mathbb{C}^{K M L / N \times K M L / N}$, therefore takes the structure of

$$
\mathbf{R}_{x x, \operatorname{sub}}=\left[\begin{array}{cccccc}
\mathbf{R}_{0,0} & \mathbf{R}_{1,0} & \mathbf{0}_{J} & \ldots & \mathbf{0}_{J} & \mathbf{R}_{K-1,0} \\
\mathbf{R}_{0,1} & \mathbf{R}_{1,1} & \mathbf{R}_{2,1} & & \mathbf{0}_{J} & \mathbf{0}_{J} \\
\mathbf{0}_{J} & \mathbf{R}_{1,2} & \mathbf{R}_{2,2} & \ddots & & \vdots \\
\vdots & & \ddots & \ddots & \ddots & \mathbf{0}_{J} \\
\mathbf{0}_{J} & & & \ddots & \mathbf{R}_{K-2, K-2} & \mathbf{R}_{K-1, K-2} \\
\mathbf{R}_{0, K-1} & \mathbf{0}_{J} & \ldots & \mathbf{0}_{J} & \mathbf{R}_{K-2, K-1} & \mathbf{R}_{K-1, K-1}
\end{array}\right]
$$

with $J=M L / N$, where

$$
\begin{aligned}
\mathbf{x}_{\mathrm{sub}}^{T}[v] & =\left[\mathbf{x}_{0}[v] \mathbf{x}_{1}[v] \cdots \mathbf{x}_{K-1}[v]\right] \text { with } \\
\mathbf{x}_{k}^{T}[v] & =\left[\mathbf{x}_{0, k}[v] \mathbf{x}_{1, k}[v] \cdots \mathbf{x}_{M-1, k}[v]\right] \\
\mathbf{x}_{m, k}^{T}[v] & =\left[\hat{x}_{m, k}[v] \hat{x}_{m, k}[v-1] \cdots \hat{x}_{m, k}[v-L / N+1]\right] .
\end{aligned}
$$

The vector $\mathbf{x}_{m, k}^{T}[v]$ contains data inside a TDL formed from the $m^{\text {th }}$ sensor signal in the $k^{\text {th }}$ subband, with $v$ being the decimated time index, $v=\frac{n}{N}$. Different from the DFT domain 
processing, the subband signals are still considered wideband — although with a reduced bandwidth as evident from Fig. 4 thus motivating the need for wideband processing in the subband domain. However, due to the reduced bandwidth note that the length of TDL for each subband channel is now approximately $N$ times shorter than the fullband case [13].

The essence for computational efficiency in subband-based beamforming is the independent processing of subband signals. Although adjacent subband signals are correlated, the off-block diagonal terms in (31) are due to redundancy in the overlap regions, and will disappear if the main block diagonal terms

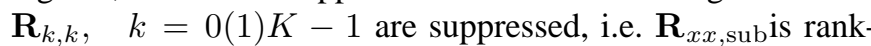
deficient. Therefore, different from critically decimated DFT domain processing, the off-block-diagonal terms in (31) can be neglected from processing without incurring a penalty, and a separate beamforming algorithm can be operated in each subband $[14,13]$.

The covariance matrix seen by the adaptive filter input is given by

$$
\mathbf{R}_{u u, \mathrm{sub}}=\mathbf{B}_{\mathrm{sb}}^{\mathrm{H}} \mathbf{R}_{x x, \mathrm{sub}} \mathbf{B}_{\mathrm{sb}}
$$

with a block diagonal blocking matrix

$$
\mathbf{B}_{\mathrm{sb}}=\left[\begin{array}{ccc}
\mathbf{B}_{0} & & \mathbf{0} \\
& \ddots & \\
\mathbf{0} & & \mathbf{B}_{K-1}
\end{array}\right]
$$

consisting of the blocking matrices $\mathbf{B}_{k} \in \mathbb{C}^{M L / N \times(M-1) L / N}$, $k=0(1) K-1$. Hence, $\mathbf{R}_{u u \text {,sub }}$ retains the structure of $\mathbf{R}_{u u, \text { sub }}$ and its redundancy. With the temporal dimension of the subband beamformers reduced by a factor of approximately $N$ and an $N$ times slower update rate due to decimation, the computational complexity of this approach accrues to

$$
\begin{gathered}
\mathcal{C}_{\mathrm{sb}}=\frac{1}{N}\left[(M+1)\left(4 K \log 2(K)+4 K+L_{p}\right)+\right. \\
\left.+K(M L / N)^{2}+K M L / N\right]
\end{gathered}
$$

MACs, whereby $L_{p}$ is the length of the filter bank's prototype and the filter bank implementation is based on a generalised DFT modulation permitting a computationally inexpensive polyphase realisation. The cost in (35) also includes the reconstruction of the beamformer output via a synthesis bank $\mathbf{G}(z)$, as indicated in Fig. 1.

\section{Performance Comparison}

\subsection{Computational Complexity}

A comparison of complexity between the various GSC implementations is provided in Fig. 5 in dependence on the temporal dimension $L$ and the number of sensor elements $M$ of the beamformer. The time domain implementation is the most costly realisation, while the lowest cost is afforded by the independent frequency bin processor in block mode. The sliding window independent frequency bin processor is more costly than the latter, but still provides a benefit in terms of complexity of overlap-save and the subband realisation, which is based

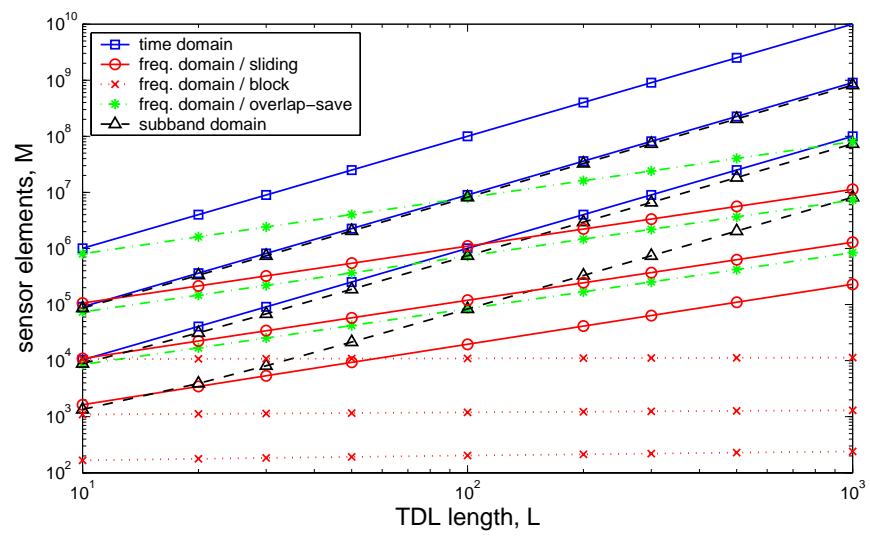

Fig. 5. Computational complexities in MACs of the various GSC implementations in dependence on the TDL length $L$ and $M=10, M=30$, and $M=100$ sensor elements each. The lowest curve reverse to $M=10$, the highest of each kind to $M=100$.

on a filter bank with $K=16$ channels decimated by $N=14$ based on a prototype with $L_{p}=448$ coefficients.

\subsection{Final MSE}

Taking the performance of the time domain beamformer as a benchmark, we assess the possible final MSE of the various implementations. Since the overlap save technique optimises the time domain cost function, its steady state performance can be expected to be similar to the time domain solution. A difference arises from the doubling of the adaptive parameters from $M L-r$ elements in $\mathbf{w}_{a}[n]$ to $2 M L-L$ elements in $\underline{w}_{a}[n]$, resulting in a potentially larger excess mean square error [21].

Block and sliding window independent frequency bin implementations suffer from the forced block-diagonalisation of the covariance matrix $\mathbf{R}_{u u \text {,ifb }}$ in (12), which is justified only if the input signal consists of frequencies coinciding with frequency bins. If the input signal violates this condition, then the MSE performance will be reduced with respect to the time domain approach. A shown in [7], potentially a rank one source could require all degrees of freedom available in the beamformer [7], and hence with the exhaustion of available degrees of freedom in the system, the steady state MSE performance can be poor.

The subband approach is based on the tri-block diagonal covariance matrix in (33). As evident from the filter bank characteristic in Fig. 4, the stopband level of the bandpass filters determines how well the idealised zero matrices $\mathbf{0}_{M L / N}$ are approximated, as they will determine the MSE bound [16]. Thus the MSE performance of the subband GSC can be controlled by the filter bank design, in contrast to sliding window and block processing methods.

\subsection{Convergence Speed}

In order to provide examples for the convergence speed of the various wideband beamformer implementations in Sec. 3, we 


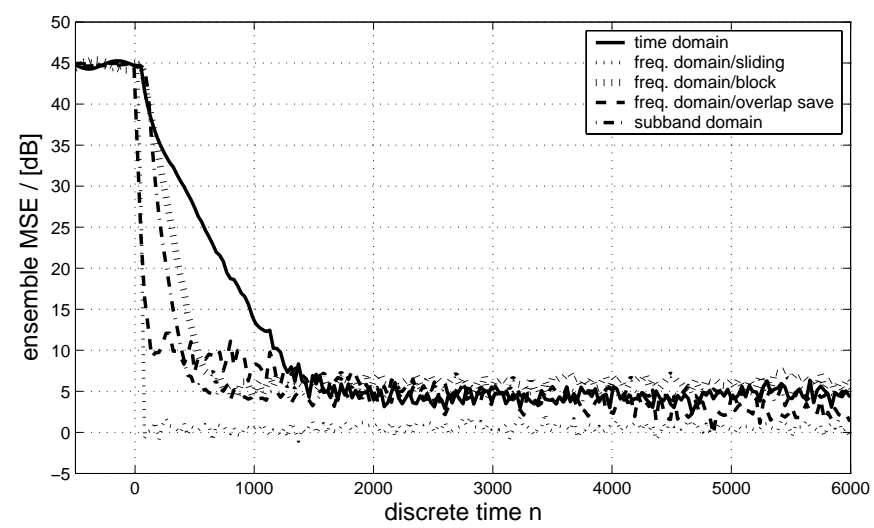

Fig. 6. Learning curves for Scenario 1.

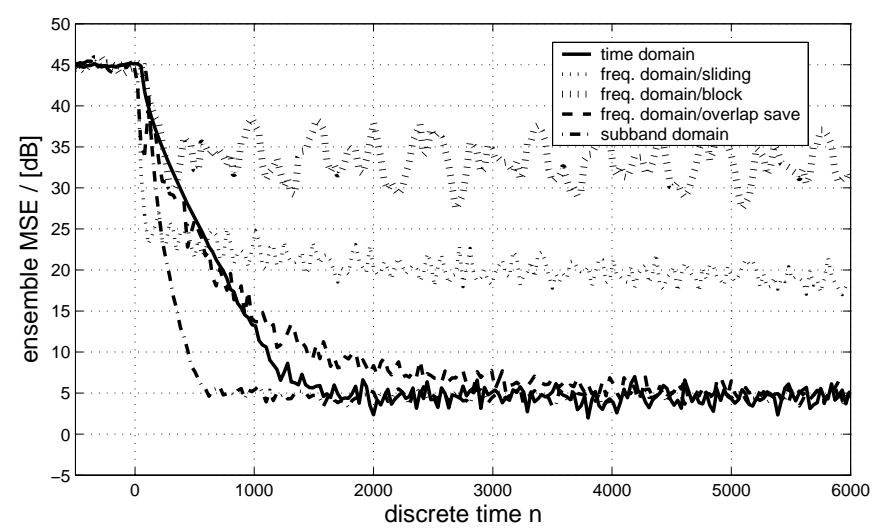

Fig. 7. Learning curves for Scenario 2.

consider an array with $M=10$ linear uniformly spaced sensors followed by TDLs of length $L=32$. In the case of a subband implementation, we utilise a filter bank with prototype length $L_{p}=96$ decomposing signals into $K=8$ subbands decimated by $N=6$. The computational complexity of the various schemes is summarised in Tab. 1. The signal of interest is at the array's broadside, while 10 narrowband jammers impinge from $-20^{\circ}$ at an SINR of $-45 \mathrm{~dB}$. Additionally, the array is corrupted by uncorrelated noise at an SNR of $-6 \mathrm{~dB}$. Below, we consider two scenarios for the interferers.

Scenario 1. All interferers sit on frequency bins, i.e. at integer multiples of $\Omega=2 \pi / L$, and not in the overlap region of the analysis filter banks of the subband implementation. The mean squared residual error (MSE), i.e. the beamformer output minus the signal of interest, over an ensemble of 100 simulations is shown in Fig. 6. For the frequency domain

Table 1. Computational cost of various beamformers.

\begin{tabular}{|l||r|r|}
\hline structure & MAC/sample & rel. cost \\
\hline time domain & 103360 & $100.00 \%$ \\
freq. domain/block & 185 & $0.18 \%$ \\
freq. domain/sliding & 5760 & $5.57 \%$ \\
freq. domain/overlap save & 416 & $0.40 \%$ \\
subband domain & 4416 & $4.27 \%$ \\
\hline
\end{tabular}

methods, the interferers sit on frequency bins and can be nulled out fast and with a single degree of freedom (DOF) - the data covariance matrix at the blocking matrix output is diagonal, and no approximation error is made by neglecting correlations between different frequency bins. The subband method converges somewhat fast than the time domain approach due to the prewhitening effect of the decomposition.

Scenario 2. All interferers lie outside the frequency bins and coincide with the overlap region of the filter banks. As can be seen in Fig. 7, the time domain algorithm is unaffected. In the subband scheme each interferer will appear in the two subbands sharing the overlap region, and two DOFs are required to suppress each rank-one interferer [7]. Since the order of the subband beamformer is large enough to provide the DOFs, the convergence characteristic is not substantially different from Fig. 6. For the DFT based approaches, block processing and sliding window methods fail due to neglecting the correlations between frequency bins. All temporal DOFs are needed to suppress a single interferer, otherwise only a limited level of attenuation can be achieved. The overlap-save method reaches a satisfactory steady-state MSE, but due to a large dynamic range in the excitation of the various frequency bins due to spectral leakage as well as block adaptation [10], convergence is slow.

\section{Conclusions}

A number of different beamformer implementations have been reviewed and compared. DFT-based techniques, in particular block processing, offer high computational savings. However, if the jammer signal components do not coincide with frequency bins, block and sliding window methods suffer from unjustified narrowband approximations. The presented overlapsave method guarantees a better steady-state value, but can incur slow convergence, while the subband approach offers a very robust performance at a somewhat higher cost, although still inexpensive and consistently faster in terms of its convergence compared to a time domain implementation.

\section{Acknowledgement}

We would like to thank Prof. Ian K. Proudler and Prof. John G. McWhirter of the Advanced Signal Processing Group, QinetiQ Ltd., Malvern, UK, for their support and technical input.

\section{References}

[1] Liuqing Yang and G. B. Giannakis, "Ultra-wideband communications: an idea whose time has come," IEEE SP Mag, 21(6): 26-54, 2004.

[2] B. D. Van Veen and K. M. Buckley, "Beamforming: A Versatile Approach to Spatial Filtering," IEEE ASSP Mag, 5(2): 4-24, 1988.

[3] R. T. Compton, "The Bandwidth Performance of a TwoElement Adaptive Array with Tapped Delay-Line Processing", IEEE Trans. AP, 36(1): 4-14, 1988. 
[4] David Nunn, "Suboptimal Frequency-Domain Adaptive Antenna Processing Algorithm for Broad-Band Environments," IEE Proc. F: Radar Sig. Proc., 134(4): 341-351, 1987.

[5] H. L. Van Trees, Optimum Array Processing, Wiley, 2002.

[6] L. C. Godara and M. R. S. Jahromi, "Limitations and Capabilities of Frequency Domain Broadband Constraint Beamforming Schemes," IEEE Trans. SP, 47(9): 23862395, 1999.

[7] S. Weiss and I. K. Proudler, "Comparing Efficient Broadband Beamforming Architectures and Their Performance Trade-Offs," in Proc. 14th Int. Conf. DSP, Santorini, Greece, 2002, 1: 417-423.

[8] W. Kellermann and H. Buchner, "Wideband Algorithms Versus Narrowband Algorithms for Adaptive Filtering in the DFT Domain," in Asilomar Conf. Sig. Sys. Comp., 2003.

[9] R. E. Crochiere and L. R. Rabiner, Multirate Digital Signal Processing, Prentice Hall, 1983.

[10] J. J. Shynk, "Frequency-Domain and Multirate Adaptive Filtering," IEEE SP Mag., 9(1): 14-37, 1992.

[11] W. Kellermann, "Analysis and Design of Multirate Systems for Cancellation of Acoustical Echoes," in Proc. IEEE ICASSP, NY, 1988, 5: 2570-2573,

[12] F. Lorenzelli, A. Wang, D. Korompis, R. Hudson, and K. Yao, "Subband Processing for Broadband Microphone Arrays," J. VLSI Sig. Proc. Sys. for Signal, Image, and Video Tech., 14(1): 43-55, 1996.

[13] S. Weiss, R. W. Stewart, M. Schabert, I. K. Proudler, and M. W. Hoffman, "An Efficient Scheme for Broadband Adaptive Beamforming," in Proc. Asilomar Conf. Sig. Sys. Comp., Pacific Grove, CA, 1999, 1: 496500 .

[14] B. Farhang-Boroujeny and Z. Wang, “Adaptive Filtering in Subbands: Design Issues and Experimental Results for Acoustic Echo Cancellation," Sig. Proc., 61(3): 213-223, 1997.

[15] L. J. Griffith and C. W. Jim, "An Alternative Approach to Linearly Constrained Adaptive Beamforming," IEEE Trans. AP, 30(1): 27-34, 1982.

[16] S. Weiss, A. Stenger, R. W. Stewart, and R. Rabenstein, "Steady-State Performance Limitations of Subband Adaptive Filters," IEEE Trans. SP, 49(9): 1982-1991, 2001.

[17] M. Harteneck, S. Weiss, and R. W. Stewart, "Design of Near Perfect Reconstruction Oversampled Filter Banks for Subband Adaptive Filters," IEEE Trans. Circ. \& Sys. II, 46(8): 1081-1086, 1999.
[18] C.-L. Koh, Broadband Adaptive Beamforming with Low Complexity and Frequency Invariant Response, technical report, Univ. of Southampton, 2004.

[19] O. L. Frost, III, “An Algorithm for Linearly Constrained Adaptive Array Processing," Proc. IEEE, 60(8): 926935, 1972.

[20] G. H. Golub and C. F. Van Loan, Matrix Computations, John Hopkins, 3rd ed., 1996.

[21] S. Haykin, Adaptive Filter Theory, Prentice Hall, 2nd ed., 1991.

[22] W. Liu, S. Weiss, and C.-L. Koh, "Constrained Adaptive Broadband Beamforming Algorithm in the Frequency Domain," in Proc. IEEE Workshop SAM, Barcelona, Spain, July 2004. 